\title{
Lied versus Oper - \\ Pole musikalischer Gattungen bei Oscar Bie
}

\author{
ELISA RINGENDAHL, TROSSINGEN
}

\section{Voraussetzungen}

Mit dem Anfangssatz seines 1913 erschienenen Buchs Die Oper sichert sich der Berliner Musikund Kunstjournalist Oscar Bie (1864-1938) bleibenden Ruhm in den einschlägigen bibliografischen Verzeichnissen. „Die Oper ist ein unmögliches Kunstwerk" ${ }^{11}$ ist nicht nur eine gewagte Aussage im Zusammenhang mit einer Gattung, über die seit Jahrhunderten theoretisiert wird, sondern sie bringt zugleich auf den Punkt, wie Bie das Wesen der Oper verstanden wissen will: Als erzwungene Einheit disparater Kunstgebiete, als "Schlachtfeld"2, das auf den Widerspruch als treibende Kraft angewiesen ist, um sich verwirklichen zu können. Bies 13 Jahre später erschienenes Buch Das deutsche Lied (1926) gibt sich moderater. Der Autor verzichtet hier auf jede Provokation, er vermeidet Problematisierungen, die die Berechtigung der Gattung Lied in Zweifel ziehen. Gerade die unterlassene Polemik ist es aber, die im Lied-Buch als Mangel erscheint. Im Gegensatz zum Opern-Buch fehlt demjenigen über das Lied die Fragestellung, an der sich eine gewinnbringende Diskussion über das Wesen der Gattung entzünden könnte. Die gegensätzliche Ausprägung der beiden Bücher scheint sich in den Verkaufszahlen niederzuschlagen. Während dasjenige über die Oper zehn Jahre nach seiner Veröffentlichung bereits in zehn Auflagen existiert, ist dem Lied-Buch weit weniger Erfolg beschieden. In den Köpfen seiner - heute gleichwohl nur noch spärlichen - Leserschaft wird Bie vorrangig mit der Gattung Oper, nicht mit der Gattung Lied assoziiert. Dafür mögen innere wie äußere Gründe verantwortlich sein: Bies Bücher dürfen aufgrund der jüdischen Herkunft ihres Autors ab 1933 nicht mehr neu aufgelegt werden, sodass dem 1926 erschienenen Lied-Buch wenig Zeit bleibt, eine breite Leserschaft anzusprechen. Zugleich fällt auf, dass die Gattung Lied Bie über Das deutsche Lied hinaus kaum zu interessieren scheint, während ein bedeutender Teil seiner Rezensionen sich mit der Oper befasst ${ }^{3}$. Die Vermutung liegt nahe, dass der Grund für dieses Missverhältnis in der Sache selbst liegt: Die Oper als Gattung, die von ihrer eigenen Paradoxie lebt, liefert Bie den Stoff für ein gelungenes Feuilleton. Das Lied, das den in der Oper wütenden Problemen fernsteht, verweigert sich diesem Zugriff.

1 Oscar Bie, Die Oper, [Berlin 1913], Reprint München etc. 1988 (Einführung zur Neuauflage von Carl Dahlhaus), S. 9. Zitiert wird hier und im Folgenden nach der Ausgabe von 1988.

2 Oscar Bie, Das Rätsel der Musik, Leipzig 1922, S. 55.

3 Bie verwertet in seinem Opern-Buch kommentarlos viele seiner früher geschriebenen Rezensionen. 
Der Titel „Lied versus Oper - Pole musikalischer Gattungen bei Oscar Bie“ deutet an, dass es darum gehen soll, sich Bies Ideen zur Musik über bestimmte Gattungsvorstellungen zu nähern. Diese Gattungsvorstellungen haben insofern ihre Brisanz, als sie - bei aller Anfechtbarkeit ihrer Prämissen - zum einen traditionelle Gattungssysteme durchbrechen können, zum anderen in Bies Denken und Schreiben über Musik unmittelbar eingreifen. Stellt man Bies problemorientierte Reflexion über die Oper seinen eher oberflächlich beschreibenden Ausführungen über das Lied gegenüber, ergibt sich die Frage, ob die Lektüre des Lied-Buches überhaupt lohnenswert ist. Anhand zweier Thesen soll erläutert werden, warum Das deutsche Lied in der BieRezeption einen wichtigen Rang einnimmt und seine Missachtung sogar zu einem verkürzten Blick auf Bies Ideen zur Musik führt.

1. Eine Gegenüberstellung beider Bücher erhellt, dass Oper und Lied bei Bie Pole seines Gattungsverständnisses darstellen. Das Lied-Buch spielt eine tragende Rolle, wenn man Bies Ideen zur Musik umfänglich ergründen will. Gleichzeitig wird an der Kluft, die Bie zwischen Lied und Oper installiert, evident, dass der Oper in Bies Gattungsverständnis eine exponierte Rolle zukommt. Das, was nach Bie das Wesen der Musik ausmacht, wird in der Oper auf die Spitze getrieben. Oper ist Musik in Reinform.

2. Die Vorstellung, die Bie jeweils von der Gattung Lied und Oper hat, greift direkt ein in die Art, wie er darüber schreibt. Die literarischen Mittel hängen von ihrem Gegenstand ab. Über die Oper lässt sich ein gutes Feuilleton schreiben, über das Lied nicht.

\section{Oper und Lied als Gattungspole}

In seinem Vorwort zum Reprint von Die Oper (1988) kommentiert Carl Dahlhaus den schlagkräftigen Anfangssatz des Werks: „Glücklich der Autor, dem es gelingt, die Idee eines Buches in eine Formel zu fassen". ${ }^{4}$ Bie bringt mit seiner These von der Oper als "unmöglichem Kunstwerk" auf den Punkt, was für ihn den Kern der Gattung ausmacht. Die Widersprüche, mit denen die Gattung ringt, zeichnen sich für ihren Reiz verantwortlich, der nicht trotzdem, sondern gerade deshalb von ihr ausgeht.

Bie versteht die Widersprüche, in die er die Oper verwickelt sieht, wörtlich. Im ersten Teil seines Buches argumentiert er, die Gattung scheitere an ihrem Anspruch, Unvereinbares zur Einheit zwingen zu wollen. Gesang, Orchester, Schauspiel, Bühnenbild und Publikum kämpften gegeneinander um die Oberhand, ohne dass der Streit zu jemandes Gunsten entschieden werde. In diesem Sinne ist die Oper für Bie eine Utopie: Sie strebt ein Ziel an, das sie nicht erreichen kann.

Im zweiten, viel umfangreicheren Teil des Buches nimmt Bie einen Querschnitt durch drei Jahrhunderte Opernkultur - von Claudio Monteverdi bis Richard Strauss - vor, erzählt aber

4 Bie, Die Oper, S. I. 
keine Geschichte der Gattung. Der Autor, der stil- wie kompositionsgeschichtlichen Kategorien mit Skepsis begegnet, glaubt nicht an den Sinn einer Darstellung, die die Geschichte der Oper als Entwicklungsgeschichte ausbreitet. Für Bie besteht, wie Dahlhaus feststellt, die treibende Kraft der Gattung aus „immer wieder neuen Versuchen, für die Widersprüche, die das Wesen der Oper ausmachen, eine Lösung zu finden, die wenigstens einen Augenblick lang gültig erscheint"

In seinem Buch Das deutsche Lied schlägt Bie einen anderen Ton an. Die Gattung wird anhand der vier Komponisten Franz Schubert, Robert Schumann, Johannes Brahms und Hugo Wolf abgehandelt. Am Rande kommen das Lied vor Schubert sowie Lieder von Richard Strauss und Max Reger zur Sprache, während Arnold Schönberg und Anton Webern mit einem knappen Absatz abgespeist werden. Bie würdigt jedes Lied-Opus mit durchschnittlich einem Satz, ohne sich auf bestimmte Kompositionen genauer einzulassen. Zwar betont er, keine überblicksartige Darstellung liefern zu wollen, aber seine flüchtige Gesamtschau vermittelt dem Leser den Eindruck eines wenig präzisen Nachschlagewerks. Seiner schwachen Argumentation zum Trotz wird durch Das deutsche Lied deutlich, dass die Gattung Lied bei Bie im größtmöglichen Kontrast zur Gattung Oper steht. Das Kriterium, auf das sich diese Feststellung stützt, ist das der von Bie selbst etablierten Widersprüchlichkeit. Bie schreibt über das Lied: „Wohin sind die Probleme geflogen, die sonst die Musik erschüttern? Ich erkenne sie wieder, aber sie sind delikat geworden und leicht und lösbar." ${ }^{\text {An }}$ anderer Stelle heißt es, das "Wunder" des Liedes sei "das rätselloseste"7. Für Bie finden sich im Lied die Konflikte, die die Oper beherrschen, beruhigt. Während die Oper an der Unvereinbarkeit ihrer Gegensätze krankt, glättet das Lied sie zur Einheit.

Bies Sicht auf das Lied folgt der konventionellen, historisch überlieferten Vorstellung von der Einheit des Ausdrucks, der Intimität und Anspruchslosigkeit der Gattung. Seine Anschauung gewinnt erst Schlagkraft, wenn sie derjenigen der Gattung Oper gegenübergestellt wird: Die Oper, maximal konfliktreich, und das Lied, maximal konfliktarm, sind Pole von Bies Gattungsverständnis. Zwischen diesen beiden Polen ordnen sich die übrigen Gattungen ein. Bies Gegenüberstellung zeitigt Folgen für sein Verständnis von Musik überhaupt:

1. Indem Bie Lied und Oper als Endpunkte einer Skala begreift, auf der die übrigen musikalischen Gattungen jeweils ihren Platz einnehmen, gewinnt er die Möglichkeit, diese Gattungen aus einer anderen Perspektive heraus in den Blick zu nehmen als in der traditionellen Gegenüberstellung von vokal und instrumental. Bie fasst Lied und Oper nicht zum Komplex der vokalen Musik zusammen, um sie durch ihr vokales Element von der Instrumentalmusik abzugrenzen. Aus der Verschiedenheit ihres Wesens entwickelt er vielmehr wichtige Kriterien für seinen Begriff von Musik.

5 Ebd., S. V.

6 Oscar Bie, Das deutsche Lied, Berlin 1926, S. 13.

7 Bie, Das Rätsel der Musik, S. 48. 
Bie, der jede Art von Systematik ablehnt, stellt kein Gattungssystem auf. Gleichwohl wirft seine Gegenüberstellung zweier vokaler Genres die Frage nach der Anordnung der instrumentalen Gattungen in diesem Entwurf auf. In seinem 1922 publizierten Essay Das Rätsel der Musik teilt Bie die für ihn in diesem Kontext relevanten Komponisten ein in "Opernnaturen" und "Antiopernnaturen"8, eine methodische Entscheidung, die vor dem Hintergrund seiner Oper-LiedPolarität konsequent ist. Dabei zählt er Johann Sebastian Bach, Ludwig van Beethoven, Robert Schumann, Johannes Brahms, Anton Bruckner und Franz Liszt zu den "Antiopernnaturen". Erklärungsbedürftig erscheint dabei vorrangig der Fall Liszt, der von Bie gerade nicht mit Richard Wagner und Strauss - der Schiene der "Neudeutschen" -, sondern mit Schumann und Brahms dem Komplex der Instrumentalmusik zugeordnet wird. Bie lässt sich auf den fingierten Gegensatz zwischen Neudeutschen und Konservativen, zwischen absoluter und Programmmusik, nicht ein. Der Terminus der absoluten Musik an sich wird von ihm sogar in Frage gestellt, indem er mit Liszt die Riege der "absoluten” Komponisten Bach, Beethoven und Brahms durchbricht. Für Bie zählt weiter auch das Oratorium zur absoluten Musik. Die Gattung wird von inm keineswegs als "Oper ohne Schauspiel" in die Nähe der Oper gerückt, sondern schlägt sich auf die Seite des Liedes, weil sie das Wort widerstandslos in der Musik aufgehen lässt. Bies Gegenüberstellung von Oper und Lied, von "Opernnatur" und "Antiopernnatur" ermöglicht es ihm, die einzelnen musikalischen Gattungen losgelöst von den Vorstellungen, die daran haften, zu betrachten. Verhärtete Dualismen wie derjenige zwischen absoluter und Programmmusik werden dadurch aufgelöst, dass Bie seinem Gattungsverständnis andere Kriterien zugrunde legt, ohne dabei die Absicht zu haben, sich auf ein neues Schema festlegen zu lassen. ${ }^{9}$

2. Bies Anschauung der Oper als äußerstes Extrem auf der Gattungsskala macht deutlich, dass er in der Oper die Eigenschaften von Musik auf die Spitze getrieben findet. In der Oper tritt das, was das Wesen der Musik ausmacht, in Reinform zutage. Diese These besagt nicht, dass das Lied weniger Musik ist als die Oper. Sie relativiert jedoch die Behauptung von der Idealität des Liedes im Gegensatz zur Widersprüchlichkeit der Oper, sofern die Voraussetzung gilt, dass das Element des Widersprüchlichen notwendig zur Musik gehört.

Der polemische Unterton, mit dem Bie in seinem Opern-Buch von der Oper als einer Gattung der Widersprüchlichkeit spricht, findet sich in dem bereits zitierten Essay von 1922 gemäßigt. Bie scheint hier "Widersprüchlichkeit" durch „Rätselhaftigkeit" zu ersetzen, während er unter letzterem das durchaus im romantischen Sinne Jenseitige, nicht Greifbare der Musik versteht. Als Exempel für diese (sich stark auf die Schriften des Musikwissenschaftlers Friedrich von Hausegger berufende) Annahme von der Transzendenz der Tonkunst führt Bie Richard Strauss' Oper Die Frau ohne Schatten an, an der für ihn das Moment der "Rätselhaftigkeit" in besonderem Maße zutage tritt. Bie verhandelt seine Idee gerade nicht an einem Werk der Instrumentalmusik, obwohl dies im Zusammenhang mit einer als metaphysisch verstandenen „Rätselhaftigkeit"

8 Bie, Das Rätsel der Musik, S. 20.

9 Die Ausführungen zu Bies Sicht auf die musikalischen Gattungen beziehen sich auf: Bie, Das Rätsel der Musik, S. $20 \mathrm{ff}$. 
näher läge. Gleichzeitig wählt Bie aber auch kein Wagnersches Musikdrama, um seine Argumentation zu stützen, etwas, was angesichts seiner Nähe zu den Gedanken Hauseggers nachvollziehbar schiene. Oper ist für Bie einerseits eine konfliktgeladene, mitunter gewaltsame Gattung, zugleich aber auch derjenige Bereich der Tonkunst, in der das Wesentliche der Musik schlechthin, das „Rätselhafte“, zum Vollzug gelangt. An der Oper wird deutlich, was Musik eigentlich ist.

\section{Der Kritiker als Künstler ${ }^{10}$}

Bie vertritt die Überzeugung, dass die schriftliche Darstellung von Kunstwerken sich ihrem Gegenstand so weit wie möglich anpassen muss. Die Form, die das Kunstobjekt vorgibt, soll sich in dem Text, der sich damit befasst, literarisch niederschlagen. Für Bie bedeutet das konkret, dass derjenige, der über Kunst schreibt, in einem anderen Medium an derselben Sache arbeitet. Seine Aufgabe als Kunstkritiker versteht Bie in dem expliziten Sinne, dass er das jeweilige Kunstobjekt in Form eines Essays reproduziert. ${ }^{11}$ Bies Ideal hat seinen historischen Ort und zeichnet ihn zunächst nicht vor seinen Zeitgenossen aus. Eine vergleichbare Zielsetzung findet sich bei dem 18 Jahre jüngeren Paul Bekker. Hans-Joachim Hinrichsen macht in einem Aufsatz ${ }^{12}$ über dessen Beethoven-Buch (1911) darauf aufmerksam, dass Bekkers Ausführungen zu Beethovens Sonate op. 31/2 nicht auf einem Kategorienfehler beruhen, wie August Halm es ihm vorwirft. Halm, der an Bekkers Darstellung bemängelt, sie vermische "poetische Beschreibungen und technische Sachverhaltserfassung" ${ }^{\prime 13}$, übersehe, so Hinrichsen, dass genau hierin Bekkers Absicht liege: Diesem gehe es gerade darum, Beethovens Werk sprachlich nachzuvollziehen. Er wolle gar nicht im strengen Sinne eine Analyse liefern, sondern mit den Mitteln, die ihm als Schriftsteller zur Verfügung stünden, die Beethoven'sche Sonate zur Erscheinung bringen. So schreibt Bekker wörtlich im Vorwort seines Buches: „Ich versuchte mich auf den Standpunkt des ausübenden Musikers zu stellen, der aus seinem persönlichen Empfinden heraus Beethovens Werke interpretiert. Nur war das Ausdrucksmittel, dessen ich mich zu bedienen hatte, nicht das Klavier, das Orchester, die menschliche Stimme, sondern das geschriebene Wort. Einzig auf diese rein künstlerische Reproduktion mittels des Wortes war mein Streben gerichtet." ${ }^{14}$

Bekker - und mit inm Bie - begreifen die Tätigkeit des Musikschriftstellers und -kritikers als künstlerische Tätigkeit. Dass Hinrichsen, der Bekkers Anspruch des sprachlichen Nachvollzugs

10 „Der Kritiker als Künstler" ist der Titel eines 1890 erschienenen Essays von Oscar Wilde.

11 Zu Bies Affinität zum Essay als angemessener Form der schriftstellerischen Reproduktion von Kunst vgl. Bie, Das Rätsel der Musik, S. 76f.; sowie Peter de Mendelssohn, S. Fischer und sein Verlag, Frankfurt a.M. 1970, S. 470-473.

12 Hans-Joachim Hinrichsen, "Ein Buch für das 20. Jahrhundert. Paul Bekkers ,Beethoven' von 1911", in: Musik und Ästhetik 77 (2016), S. 7-24.

13 Ebd., S. 9.

14 Zit. nach: Hinrichsen. „Ein Buch für das 20. Jahrhundert”, S. 10. 
als einen "für ein Buch überaus ungewöhnlichen"15 bezeichnet, Bie mit keinem Wort erwähnt, fällt angesichts der Tatsache auf, dass gerade Bie dieses Verfahren mit äußerster Konsequenz verfolgt. Gleichwohl sind Bies Resultate nicht alle gleich überzeugend. So bleibt sein Versuch des sprachlichen Nachvollzugs von Musik blass, wenn er sich wie in Das deutsche Lied nur auf platt Formales bezieht: Bie möchte sich der Form des Liedes schreibend anpassen, indem er sein Buch aus zahlreichen kurzen Kapiteln zusammenstellt, die jedes für sich eine geschlossene Einheit bilden und gewollt willkürlich angeordnet sind. Der Tonfall scheint sich demjenigen in einer Art freien Fantasie annähern zu wollen, wobei der Eindruck erzeugt werden soll, Bie säße gerade beim Primavista-Spiel am Klavier und zeichne aus einer besonderen Stimmung heraus seine Gedanken auf. Die intime Häuslichkeit, die Bie der Gattung Lied anheftet, versucht er sprachlich nachzuzeichnen, nicht ohne dass ihm dabei manche sentimentale Entgleisung unterläuft.

In Die Oper gelingt das Verfahren besser. Bie, der viele seiner bereits früher geschriebenen Opernkritiken kommentarlos aneinanderreiht, verfolgt das Ziel, durch das unbegründete Nebeneinander voneinander unabhängiger Texte den Eindruck des "Schlachtfeldes" zu erzeugen, als das er die Oper sieht. Am Ende dieser Anstrengung steht ein großes, aber in sich disparates Ganzes, das aus zahllosen Paradoxien erwächst. Die Widersprüchlichkeit, die Bie als treibende Kraft der Gattung betrachtet, greift in die literarische Darstellung ein: Der Schreibstil im OpernBuch entzündet sich an dem, was den Gegenstand ausmacht. Im Lied-Buch dagegen fruchtet das Verfahren nicht, weil es an einer Problemstellung mangelt, an der Bies sprachlicher Nachvollzug sich abarbeiten könnte. Vor diesem Hintergrund erstaunt es nicht, dass Bie im Laufe seines Lebens zahllose Texte über die Oper verfasst hat, während sich seine Beschäftigung mit der Gattung Lied in einem einzigen Buch erschöpft.

\section{Ausblick}

Die wenigen Ansätze einer musik-, kunst- oder literaturwissenschaftlichen Rezeption von Bies Schriften, die es bis heute gibt, stempeln den Autor zum Vertreter einer "impressionistischen" Kunstkritik des Kaiserreichs und der Weimarer Republik. ${ }^{16}$ Sofern man seinen humorigen Skeptizismus gegenüber stil- und gattungsgeschichtlichen Kategorien nicht gar als bloße Allüre abtut, gilt Bie als geschmäcklerischer Ästhetizist, der sämtliche Erscheinungen des geistigen Lebens dem subjektiven Eindruck unterwirft und Fakten nicht gelten lässt. ${ }^{17}$ Dass es Bie bei

15 Ebd.

16 Vgl. hierzu: Richard Hamann und Jost Hermand, Epochen deutscher Kultur von 1870 bis zur Gegenwart, Bd. 3, Impressionismus, München 1974; Volker Kalisch, Art. „Oscar Bie”, in: Die Musik in Geschichte und Gegenwart. Allgemeine Enzyklopädie der Musik, 2., neubearbeitete Ausgabe, hrsg. von Ludwig Finscher, Personenteil, Bd. 2, Kassel etc. 1996, Sp. 1592-1593; Wolfgang Grothe, „Die Neue Rundschau des Verlages S. Fischer. Ein Beitrag zur Publizistik und Literaturgeschichte der Jahre von 1890 bis 1925", in: Archiv für Geschichte des Buchwesens Bd. IV, Frankfurt a.M. 1963, Sp. 809-1569, Sp. 858, 862.

17 Vgl. hierzu: Richard Hamann und Jost Hermand, Epochen deutscher Kultur von 1870 bis zur Gegenwart. 
allem vermeintlichen Subjektivismus darum zu tun ist, über das Wesen der Kunst triftige Aussagen zu machen, wird übersehen. Der Ausgangspunkt des eigenen ästhetischen Empfindens als entscheidende Instanz, den Bie wählt, wenn er in seinen Texten eine betont künstlerische Herangehensweise pflegt, ist Teil seines Denkens über das Verhältnis von Kunstwerk und Kunstbetrachter. Er entspringt der Überzeugung, dass dieser Weg die Möglichkeit der Erkenntnis in den Prozess der Kunsterfahrung enthält.

Die "anti-systematische" Methode, die Bie unterstellt wird, ohne dass jenseits von ZeitgeistArgumenten darüber nachgedacht würde, welchen Nutzen Bie aus ihr zieht, sorgt in Die Oper nicht nur gattungsbezogen für erhellende Einsichten, sondern kann auch für die Musikhistoriografie insgesamt fruchtbar werden: Indem Bie sich dagegen entscheidet, die Geschichte der Oper als Entwicklungsgeschichte zu erzählen, hält er sich die Möglichkeit offen, sich immer neu zum ästhetischen Gegenstand zu verhalten. Musikalische Werke sind demgemäß keine Stationen einer fortlaufenden Gattungsgeschichte, sondern erfinderische Lösungsversuche gattungsspezifischer Probleme. ${ }^{18}$

Zitation: Elisa Ringendahl, "Lied versus Oper - Pole musikalischer Gattungen bei Oscar Bie”, in: Freie Beiträge zur Jahrestagung der Gesellschaft für Musikforschung 2019, hrsg. von Nina Jaeschke und Rebecca Grotjahn (= Musikwissenschaft: Aktuelle Perspektiven. Bericht über die Jahrestagung der Gesellschaft für Musikforschung 2019 in Paderborn und Detmold, Bd. 1), Detmold 2020, S. 292-299, DOI: 10.25366/2020.70.

18 Dies stellt Carl Dahlhaus in den fünf Seiten seines Vorworts zu Die Oper erhellend dar. 


\section{Abstract}

A comparison of Oscar Bie's monographies Die Oper and Das deutsche Lied shows that opera and 'Lied' are two extremes in Bie's understanding of musical genres. While opera according to Bie is an "impossible work of art" which arises from countless contradictions, the low-conflict 'Lied' forms an opposite pole. Bie's perception influences the way he deals with the two genres in writing: while opera is suitable for an adequate feuilleton, 'Lied' is not. Bie's comparison gives him the possibility to break with established patterns of genres. It becomes apparent that for him opera takes on a special role in that it represents the culmination of the basic features of music. As a critic, Bie demands utmost congruence between object and written representation. Writing about music means to be an artist who deals with the same work of art but in another medium.

\section{Kurzvita}

Elisa Ringendahl, geboren 1989, studierte Klavier und Musikwissenschaft an der Musikhochschule Trossingen und schloss beide Fächer mit einem Master ab. Neben ihrer publizistischen Tätigkeit für verschiedene Fachzeitschriften konzertiert sie regelmäßig und unterrichtet als Klavierpädagogin an der Musikschule Winterthur. Derzeit promoviert sie an der Musikhochschule Trossingen über den Musik- und Kunstpublizisten Oscar Bie. 


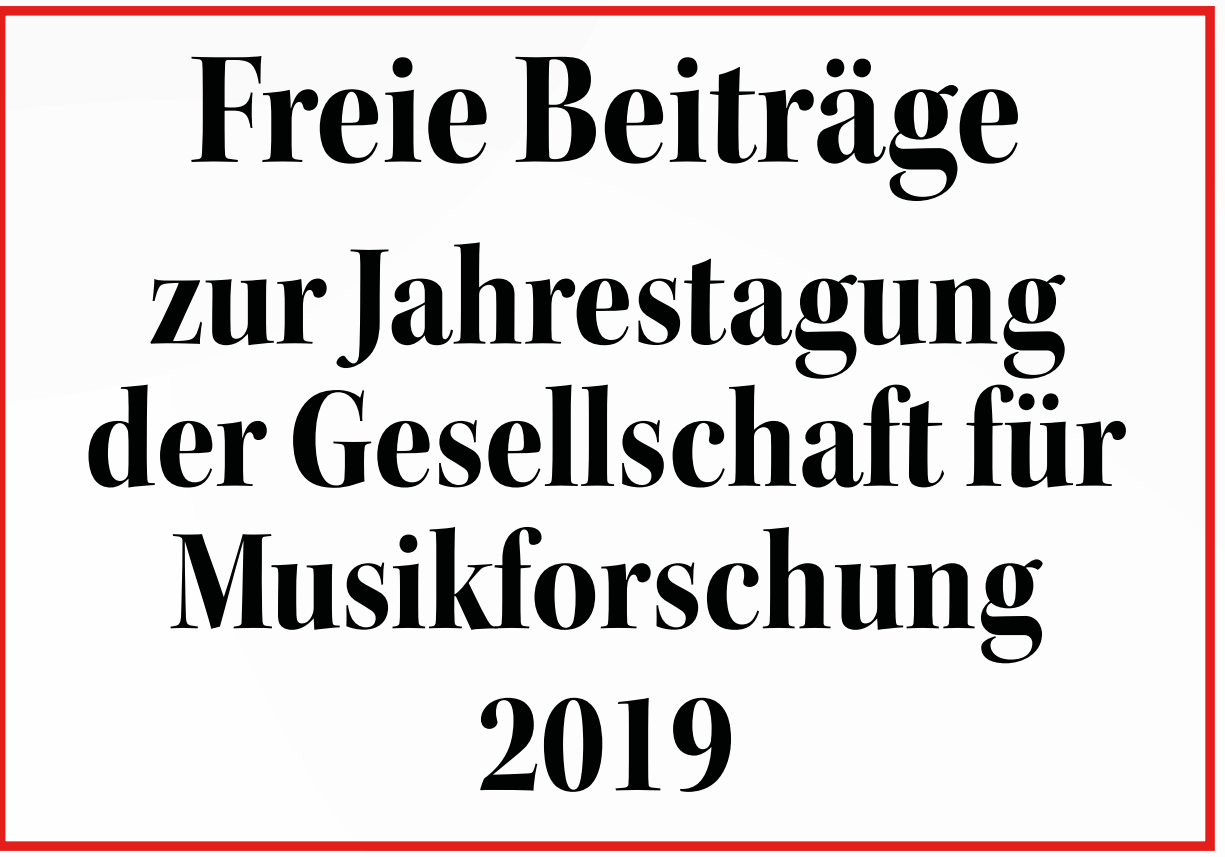

Herausgegeben von Nina Jaeschke und Rebecca Grotjahn

Musikwissenschaft: Aktuelle Perspektiven 1 
Freie Beiträge 


\section{Musikwissenschaft: Aktuelle Perspektiven}

Bericht über die Jahrestagung der Gesellschaft für Musikforschung 2019 in Paderborn und Detmold

Herausgegeben von Rebecca Grotjahn und Nina Jaeschke

Band 1 


\section{Freie Beiträge}

\section{zur Jahrestagung der Gesellschaft für Musikforschung 2019}

Herausgegeben von Nina Jaeschke und Rebecca Grotjahn

Detmold: Musikwissenschaftliches Seminar der Universität Paderborn und der Hochschule für Musik Detmold 2020 
DOI: $10.25366 / 2020.42$

Online-Version verfügbar unter der Lizenz: Urheberrecht 1.0, $<$ https://rightsstatements.org/page/InC/1.0/?language=de>

Bibliografische Information der Deutschen Nationalbibliothek

Die Deutsche Nationalbibliothek verzeichnet diese Publikation in der Deutschen Nationalbibliografie; detaillierte bibliografische Daten sind im Internet über http://dnb.d-nb.de abrufbar.

\section{Impressum}

Redaktion: Nina Jaeschke, Rebecca Grotjahn und Jonas Spieker Satz: Nina Jaeschke

(C) Musikwissenschaftliches Seminar der Universität Paderborn und der Hochschule für Musik Detmold 2020 


\section{INHALT}

Vorwort $\quad$ IX

Komponieren für das Radio: Akteure, Diskurse, Praktiken $\quad 1$

Musikwissenschaft - Feminismus - Kritik: Ein Generationenaustausch 6

\section{Stefan Alschner}

Der Wagner-Sänger Joseph Aloys Tichatschek - Vom Nachlass zum Netzwerk

\section{Alenka Barber-Kersovan}

Songs for the Goddess. Das popmusikalische Neo-Matriarchat zwischen Ethno-Beat,

erfundenen Traditionen und kommerzieller Vermarktung

Elias Berner, Julia Jaklin, Peter Provaznik, Matej Santi, Cornelia Szabó-Knotik

Musikgeschichte anders erzählen? Das Beispiel der 1970er in Österreich.

Musikhistoriographie in der Zeit der Digitalisierung

\section{Mauro Fosco Bertola}

„Ein Laut so klagevoll”. Lohengrin zwischen Richard Wagner und Salvatore Sciarrino

\section{Matthieu Cailliez}

Europäische Rezeption der Berliner Hofoper und Hofkapelle von 1842 bis 1849

\section{lacopo Cividini}

Zwischen klassischer Musikphilologie und angewandter Informatik:

Die Digitale Mozart-Edition (DME) der Stiftung Mozarteum Salzburg

\section{Marko Deisinger}

Fortschrittliche Technologie im Dienste eines Antimodernisten.

Heinrich Schenker und der österreichische Rundfunk

\section{Norbert Dubowy}

Vom Kritischen Bericht zur Kritischen Dokumentation am Beispiel der Digital-interaktiven Mozart-Edition

\section{Markus Engelhardt}

Musik zwischen Nation Building und Internationalität. Italien um 1900

\section{Maryam Haiawi}

Das Oratorium im Spannungsfeld der Konfessionen: 


\section{Judith I. Haug}

"Manch eine*r liegt, morgens noch trunken, im Rosengarten" - Rekonstruktionen

osmanischer Musikgeschichte in Gesangstextsammlungen

\section{Renate Koch}

Marcel Prawy und das erste Broadway-Musical im Österreich der Nachkriegszeit

Susanne Kogler, Julia Mair, Juliane Oberegger, Johanna Trummer

Erich Marckhl - Musikausbildung in der Steiermark nach 1945.

Brüche und Kontinuitäten

\section{Marie-Anne Kohl}

Die weinende Jury. "Geschlechtslose" Tränen bei globalen Musik-Castingshows?

\section{Fabian Kolb}

Tanztheater und filmische Ästhetik. Cineastische Einflüsse und Gestaltungsweisen in den Kompositionen für die Ballets Suédois 1920-1925

\section{Christian Lehmann}

Tempobezeichnungen von Julius Stockhausen für Die schöne Müllerin:

Ein Quellenfund

\section{Martin Link}

Signum et gens - Zur Gendersemiotik in Clara und Robert Schumanns Liederzyklus Liebesfrühling

\section{Livio Marcaletti}

„Strafspiel" und satirische Stilmittel in musikdramatischen Gattungen des frühen 18. Jahrhunderts

\section{Tobias Marx, Martin Lissner}

Thüringer Musikszene - Jugendmusikredaktionen als außerschulische musikbezogene Bildungskontexte

\section{Maho Naito}

Die Parallelität der Entstehungsprozesse der ersten beiden Symphonien Gustav

Mahlers: Instrumentation, Revision und Dirigierpraxis

\section{Elisa Novara}

Eine Schumann-Werkstatt? Zur Übertragbarkeit der Methoden vom Projekt 
Theodora Oancea, Joachim Pollmann, Jonas Spieker

Kollaborateure - Involvierte - Profiteure. Erarbeitung eines Online-Lexikons zur

Musik in der NS-Zeit

\section{Kiron Patka}

„Ich wollte eigentlich Sängerin werden." Berufsselbstbilder von Tontechniker*innen im Radio

\section{Siegwart Reichwald}

Die Leiden der jungen Clara: Das Klaviertrio Opus 17 als Ausdruck einer Neu-

Romantikerin

\section{Elisa Ringendahl}

Lied versus Oper - Pole musikalischer Gattungen bei Oscar Bie

\section{Benedikt Schubert}

Struktur und Exegese. Über Eigentümlichkeiten in der Arie "Des Vaters Stimme ließ sich hören" (BWV 7/4)

Uwe Seifert, Sebastian Klaßmann, Timo Varelmann, Nils Dahmen

Computational Thinking in der Musikwissenschaft: Jupyter Notebook als Umgebung

für Lehre und Forschung

\section{Yusuke Takamatsu}

Synthese als Modus der Prozessualität bei Schubert:

Sein spezifisches Wiederholungsprinzip im langsamen Satz

\section{Daniel Tiemeyer}

Johann Nepomuk Hummels Sonate in fis-Moll Op. 81 - Studien zu Entstehungs-

hintergrund, Rezeption und formalerStruktur

\section{Andrea van der Smissen}

Musikalische Innovation im Umfeld der Moderne und historischen Avantgarde in Ungarn

\section{Tim Ziemer, Holger Schultheis}

Psychoakustische Sonifikation zur Navigation in bildgeführter Chirurgie

\section{Magdalena Zorn}

Musik mit dem Radio hören: Über den Begriff der musikalischen Aufführung 
Gabriele Buschmeier in memoriam 


\section{Vorwort}

Die vorliegenden Bände dokumentieren die Jahrestagung der Gesellschaft für Musikforschung 2019. In den dreieinhalb Tagen vom 23. bis zum 26. September 2019 wurden in Paderborn und Detmold nicht weniger als 185 Beiträge präsentiert, verteilt auf diverse Symposien, Round tables, Freie Sektionen und Postersessions. Sie alle auf einen Nenner bringen zu wollen, ist ein Ding der Unmöglichkeit - und das ist gut so, ist es doch Ziel der Jahrestagungen, die große Vielfalt der Themen und Methoden des Faches Musikwissenschaft abzubilden. Um die thematische Vielfalt der freien Referate angemessen abbilden zu können und gleichzeitig den inhaltlichen Schwerpunkten der beiden hier publizierten Hauptsymposien ausreichend Raum bieten zu können, erscheinen diese in drei Bänden.

„Musikwissenschaft: Aktuelle Perspektiven": Der Titel der kleinen Reihe ist keine Verlegenheitslösung. Musikwissenschaft im Kontext der Digital Humanities; Musikwissenschaft und Feminismus; Musik und Medien; Musikalische Interpretation - schon die Felder, die von den vier Hauptsymposien bespielt wurden, wären noch vor wenigen Jahrzehnten allenfalls an der Peripherie das Faches zu finden gewesen. Sie entsprechen Arbeitsschwerpunkten der Lehrenden am Musikwissenschaftlichen Seminar der Universität Paderborn und der Hochschule für Musik Detmold, das die Tagung ausrichtete. Zugleich nehmen sie Bezug auf aktuelle Ereignisse und Entwicklungen. So erwuchs das von Andreas Münzmay und Joachim Veit organisierte Symposium „Brückenschläge - Informatik und Musikwissenschaft im Dialog" unmittelbar aus den Erfahrungen im Virtuellen Forschungsverbund Edirom (ViFE) und im fakultäten- und hochschulübergreifenden Zentrum Musik-Edition-Medien (ZenMEM). Der 200. Geburtstag von Clara Wieck/Schumann war der Anlass für das von Rebecca Grotjahn geleitete Symposium „Die Begleiterin - Clara Schumann, Lied und Liedinterpretation", das in enger Kooperation mit der Hochschule für Musik Detmold durchgeführt wurde. Das Hauptsymposium „Brückenschläge" wird in einem separaten Band publiziert (Bd. 3 der vorliegenden Reihe). Im Rahmen dieses Symposiums führte die von Stefanie Acquavella-Rauch geleitete Fachgruppe Digitale Musikwissenschaft eine Posterpräsentation durch, die von den Beiträger*innen erfreulicherweise zu kürzeren Texten umgearbeitet wurden, sodass sie hier ebenfalls, zusammen mit den Postern,

publiziert werden können. Hinzu kommen einige Beiträge, die bereits bei der Jahrestagung 2018 in Osnabrück präsentiert wurden. Auch das Hauptsymposium "Die Begleiterin" wird in einem eigenen Band (Bd. 2) publiziert. Die Beiträge zu den beiden anderen Hauptsymposien hingegen werden an anderen Orten veröffentlicht; in Band 1 („Freie Beiträge zur Jahrestagung der Gesellschaft für Musikforschung 2019") der vorliegenden Publikation finden sich jedoch Einführungen und Abstracts. Das Symposium „Komponieren für das Radio" unter Leitung von Antje Tumat und Camilla Bork (Katholieke Universiteit Leuven) behandelte Einflüsse des Mediums auf Kompositionsprozesse sowie durch radiophone Kompositionen bzw. radiophonen Klang ausgelöste Diskurse. Sarah Schauberger und Cornelia Bartsch (Universität Oldenburg) nahmen das 25-jährige Jubiläum der Fachgruppe Frauen- und Genderstudien zum Anlass für einen Generationenaustausch zum Thema "Musikwissenschaft - Feminismus - Kritik": Was wa- 
ren vor einem Vierteljahrhundert Inhalte und Aufgaben einer feministischen Musikwissenschaft und wie kann sich diese heute positionieren?

Bewusst haben wir im Tagungsbericht auf inhaltliche Eingriffe in die Beiträge verzichtet. ${ }^{1}$ Das gilt besonders für die Freien Referate: Es galt, den Charakter der Jahrestagung als Forum für ,freie', d. h. innovative und auch experimentelle Gedanken zu wahren. Einige Kolleg*innen, die die Tagung mit Vorträgen und Posterpräsentationen bereichert hatten, haben sich gegen eine Publikation im vorliegenden Band entschieden - sei es, weil sie eine Möglichkeit fanden, ihre Beiträge in einem inhaltlich passenderen Rahmen zu veröffentlichen, sei es, weil ihre Überlegungen in ihre entstehenden Qualifikationsschriften fließen sollen, oder sei es, weil sie von den Autor*innen in der vorgetragenen Form zunächst verworfen wurden. Auch damit erfüllt eine Freie-Referate-Sektion ihren Zweck: Die Diskussionen mit der versammelten Fach-Öffentlichkeit sollen dabei helfen, Gedanken weiterzuentwickeln und zu verändern. In diesem Sinne sei allen Beteiligten - den Autor*innen, den nichtpublizierenden Referent*innen und den Mit-Diskutant*innen - ganz herzlich gedankt für ihr Mitwirken bei der Tagung.

Unser herzlicher Dank gilt einer Reihe weiterer Personen, die zum Gelingen dieser drei Bände beigetragen haben. Hier ist besonders Jonas Spieker zu nennen, der uns tatkräftig bei der Redaktion geholfen hat. Andrea Hammes (SLUB Dresden) sei herzlich für die Aufnahme unseres Bandes auf musiconn.publish gedankt - wir freuen uns, damit unsererseits zur Etablierung dieser innovativen Publikationsplattform beizutragen.

Erneut möchten wir an dieser Stelle allen Menschen danken, die uns bei der Organisation, Ausrichtung und Finanzierung der Tagung selbst unterstützt haben: der Präsidentin der Universität Paderborn, Prof. Dr. Birgitt Riegraf, dem Rektor der Hochschule für Musik Detmold, Prof. Dr. Thomas Grosse, den Kolleginnen und Kollegen der beiden beteiligten Hochschulen, dem Vorstand der Gesellschaft für Musikforschung, der Universitätsgesellschaft Paderborn und allen Sponsoren. Besonders dankbar sind wir den Mitarbeiter*innen und den studentischen bzw. wissenschaftlichen Hilfskräften des Musikwissenschaftlichen Seminars, die bei der Vorbereitung und Ausrichtung der Tagung immensen Einsatz zeigten - stellvertretend sei an dieser Stelle Johanna Imm erwähnt, die zusammen mit Nina Jaeschke das Herz des Organisationsteams bildete.

Wir widmen diese Reihe Dr. Gabriele Buschmeier, dem langjährigen Vorstandsmitglied der Gesellschaft für Musikforschung, die kurz vor der Publikation dieses Bandes unerwartet verstarb.

Detmold, im September 2020

Rebecca Grotjahn und Nina Jaeschke

Zitation: Rebecca Grotjahn und Nina Jaeschke, „Vorwort”, in: Freie Beiträge zur Jahrestagung der Gesellschaft für Musikforschung 2019, hrsg. von Nina Jaeschke und Rebecca Grotjahn (= Musikwissenschaft: Aktuelle Perspektiven. Bericht über die Jahrestagung der Gesellschaft für Musikforschung 2019 in Paderborn und Detmold, Bd. 1), Detmold 2020, S. IX-X, DOI: 10.25366/2020.43.

1 Freigestellt war den Autor*innen auch, ob sie sich für eine gendersensible Sprache entscheiden bzw. welche Form des Genderns sie bevorzugen. 